\title{
Dataset of "true mangroves" plant species traits
}

\author{
Aline Ferreira Quadros ${ }^{\ddagger}$, Martin Zimmer ${ }^{\ddagger}$ \\ ‡ Leibniz Centre for Tropical Marine Research, Bremen, Germany
}

Corresponding author: Aline Ferreira Quadros (quadros.af@gmail.com)

Academic editor: Luis Cayuela

Received: 06 Nov 2017 | Accepted: 29 Nov 2017| Published: 29 Dec 2017

Citation: Quadros A, Zimmer M (2017) Dataset of "true mangroves" plant species traits. Biodiversity Data Journal 5: e22089. https://doi.org/10.3897/BDJ.5.e22089

\section{Abstract}

\section{Background}

Plant traits have been used extensively in ecology. They can be used as proxies for resource-acquisition strategies and facilitate the understanding of community structure and ecosystem functioning. However, many reviews and comparative analysis of plant traits do not include mangroves plants, possibly due to the lack of quantitative information available in a centralised form.

\section{New information}

Here a dataset is presented with 2364 records of traits of "true mangroves" species, gathered from 88 references (published articles, books, theses and dissertations). The dataset contains information on 107 quantitative traits and 18 qualitative traits for 55 species of "true mangroves" (sensu Tomlinson 2016). Most traits refer to components of living trees (mainly leaves), but litter traits were also included.

\section{Keywords}

Mangroves, Rhizophoraceae, leaf traits, plant traits, halophytes 


\section{Introduction}

The vegetation of mangrove forests is loosely classified as "true mangroves" or "mangrove associates". True mangroves are woody plants, facultative or obligate halophytes (Wang et al. 2011). "True mangroves" are defined by Tomlinson (2016) as plant species that 1) occur only in mangrove forests and are not found in terrestrial communities; 2) play a major role in the structure of the mangrove community, sometimes forming pure stands; 3) have morphological specialisations to the mangrove environment; 4) have some mechanism for salt exclusion. Other notable specialisations of mangrove plants include: aerial roots to counteract the anaerobic sediments, support structures such as buttresses and aboveground roots, low water potentials and high intracellular salt concentrations, salt-excretion through leaves and buoyant, viviparous propagules (Duke et al. 1998).

Following Tomlinson (2016), all species of genera Avicennia, Lumnitzera, Bruguiera, Ceriops, Kandelia, Rhizophora and Sonneratia, plus the species Nypa fruticans and Laguncularia racemosa, are considered as "true mangroves" and are the major components of mangrove forests worldwide. Other species, such as Acrostichum aureum, Aegiceras corniculatum, Osbornia octodonta et al., are also "true mangroves" but considered as minor components of mangrove forests (Tomlinson 2016).

Mangrove forests are highly threatened worldwide (Duke et al. 2007) and conservation efforts face the lack of a good understanding of mangrove community structure and ecosystem processes. With this gap in mind, literature on mangrove trees was reviewed and a dataset of traits was assembled, with the aim of contributing to future studies of mangroves using a functional trait perspective and also to allow the inclusion of mangrove trees in future comparative studies of plant ecology and resource-acquisition strategies.

\section{Geographic coverage}

Description: Global

\section{Taxonomic coverage}

Description: This dataset contains traits for 55 species of "true mangroves". To standardise the spelling of species' names, The Plant List (2013) was followed. Some species listed below are currently considered as synonyms in The Plant List (e.g. Avicennia alba is currently a synonym of Avicennia marina). However, they were chosen to be included under the names given by the authors to allow the tracking of the original information. All records of Ceriops tagal var. australis were included as Ceriops australis, and Ceriops tagal var. tagal was included as Ceriops tagal following Ballment et al. (1988). 
Taxa included:

\begin{tabular}{|c|c|}
\hline Rank & Scientific Name \\
\hline species & Acanthus ilicifolius \\
\hline species & Acrostichum aureum \\
\hline species & Aegialitis annulata \\
\hline species & Aegialitis rotundifolia \\
\hline species & Aegiceras corniculatum \\
\hline species & Avicennia alba \\
\hline species & Avicennia bicolor \\
\hline species & Avicennia eucalyptifolia \\
\hline species & Avicennia germinans \\
\hline species & Avicennia integra \\
\hline species & Avicennia lanata \\
\hline species & Avicennia marina \\
\hline species & Avicennia officinalis \\
\hline species & Avicennia rumphiana \\
\hline species & Avicennia schaueriana \\
\hline species & Bruguiera cylindrica \\
\hline species & Bruguiera exaristata \\
\hline species & Bruguiera gymnorhiza \\
\hline species & Bruguiera hainesii \\
\hline species & Bruguiera parviflora \\
\hline species & Bruguiera rhynchopetala \\
\hline species & Bruguiera sexangula \\
\hline species & Camptostemon schultzii \\
\hline species & Ceriops australis \\
\hline species & Ceriops decandra \\
\hline species & Ceriops tagal \\
\hline species & Excoecaria agallocha \\
\hline species & Kandelia candel \\
\hline species & Kandelia obovata \\
\hline
\end{tabular}




\begin{tabular}{|c|c|}
\hline species & Laguncularia racemosa \\
\hline species & Lumnitzera littorea \\
\hline species & Lumnitzera racemosa \\
\hline species & Nypa fruticans \\
\hline species & Osbornia octodonta \\
\hline species & Pelliciera rhizophorae \\
\hline species & Rhizophora apiculata \\
\hline species & Rhizophora harrisonii \\
\hline species & Rhizophora lamarckii \\
\hline species & Rhizophora mangle \\
\hline species & Rhizophora mucronata \\
\hline species & Rhizophora racemosa \\
\hline species & Rhizophora samoensis \\
\hline species & Rhizophora stylosa \\
\hline species & Scyphiphora hydrophylacea \\
\hline species & Sonneratia alba \\
\hline species & Sonneratia apetala \\
\hline species & Sonneratia caseolaris \\
\hline species & Sonneratia griffithii \\
\hline species & Sonneratia gulngai \\
\hline species & Sonneratia hainanensis \\
\hline species & Sonneratia lanceolata \\
\hline species & Sonneratia ovata \\
\hline species & Xylocarpus granatum \\
\hline species & Xylocarpus mekongensis \\
\hline species & Xylocarpus moluccensis \\
\hline
\end{tabular}

\section{Traits coverage}

This dataset contains 18 qualitative traits (Table 1) and 107 quantitative traits (Table 2). The number of records per species and trait is shown in Suppl. material 1. The number of traits available per species varies from 2 to 95 and is shown in Fig. 1. 


\section{Table 1.}

Detailed list of qualitative traits and respective references.

\begin{tabular}{|c|c|c|c|}
\hline Trait name & $\begin{array}{l}\text { Type of } \\
\text { information }\end{array}$ & Possible values & References \\
\hline $\begin{array}{l}\text { dispersal unit floating } \\
\text { capacity in freshwater }\end{array}$ & categorical & $\begin{array}{l}\text { floater; } \\
\text { sinker }\end{array}$ & Clarke et al. 2001 \\
\hline $\begin{array}{l}\text { dispersal unit floating } \\
\text { capacity in saltwater }\end{array}$ & categorical & $\begin{array}{l}\text { floater; } \\
\text { sinker }\end{array}$ & $\begin{array}{l}\text { Clarke et al. 2001, Giesen et } \\
\text { al. } 2007\end{array}$ \\
\hline $\begin{array}{l}\text { dispersal unit } \\
\text { orientation in water }\end{array}$ & categorical & prone; prone to vertical; vertical & Clarke et al. 2001 \\
\hline dispersal unit shape & categorical & $\begin{array}{l}\text { tear-drop; ovoid, round; long curved; long; } \\
\text { ellipsoidal; obovate; flattened-round }\end{array}$ & Giesen et al. 2007 \\
\hline $\begin{array}{l}\text { dispersal unit size } \\
\text { class }\end{array}$ & ordinal & $\begin{array}{l}\mathrm{I}=<0.1 \mathrm{~cm}^{3} \\
\mathrm{II}=<1 \mathrm{~cm}^{3} \\
\mathrm{III}=<10 \mathrm{~cm}^{3} \\
\mathrm{IV}=<100 \mathrm{~cm}^{3} \\
\mathrm{~V}=<1000 \mathrm{~cm}^{3}\end{array}$ & Duke et al. 1998 \\
\hline germination type & categorical & $\begin{array}{l}\text { epigeal; } \\
\text { hypogeal }\end{array}$ & $\begin{array}{l}\text { Clarke et al. 2001, Soepadmo } \\
\text { et al. 2002, Tomlinson } 1986\end{array}$ \\
\hline $\begin{array}{l}\text { leaf emergences } \\
\text { (pubescence) }\end{array}$ & binary & $\begin{array}{l}\text { yes; } \\
\text { no }\end{array}$ & $\begin{array}{l}\text { Giesen et al. 2007, NParks } \\
\text { 2017, Reef and Lovelock } \\
\text { 2015, Sheue et al. } 2003\end{array}$ \\
\hline plant growth form & categorical & $\begin{array}{l}\text { shrub/small tree; } \\
\text { tree }\end{array}$ & Giesen et al. 2007 \\
\hline $\begin{array}{l}\text { plant position in the } \\
\text { intertidal }\end{array}$ & ordinal & $\begin{array}{l}\mathrm{L}=\text { low; } \\
\mathrm{M}=\text { mid; } \\
\mathrm{H}=\text { high; } \\
\mathrm{ML}=\text { middle to low; } \\
\mathrm{HM} \text { = high to middle; } \\
\mathrm{HML} \text { = high, middle and low }\end{array}$ & Clough 1992, Duke et al. 1998 \\
\hline $\begin{array}{l}\text { plant preferred } \\
\text { substrate }\end{array}$ & categorical & $\begin{array}{l}\text { Sand; clay; mud; riverbanks; mud/sand/ } \\
\text { peaty soils; mudflat/sand/calcareous; } \\
\text { sand/mud; soft fine-grained; }\end{array}$ & Giesen et al. 2007 \\
\hline $\begin{array}{l}\text { plant tolerance to } \\
\text { drought }\end{array}$ & ordinal & $\begin{array}{l}1 \text { = very low; } \\
2 \text { = low; } \\
3 \text { = mid; } \\
4 \text { = high; } \\
5 \text { = very high; }\end{array}$ & Clough 1992 \\
\hline $\begin{array}{l}\text { plant tolerance to low } \\
\text { temperature }\end{array}$ & ordinal & $\begin{array}{l}1 \text { = very low; } \\
2 \text { = low; } \\
3 \text { = mid; } \\
4 \text { = high; } \\
5 \text { = very high; }\end{array}$ & Clough 1992 \\
\hline
\end{tabular}




\begin{tabular}{|l|l|l|l|}
\hline plant tolerance to salt & ordinal & $\begin{array}{l}1=\text { very low; } \\
2=\text { low; } \\
3=\text { mid; } \\
4=\text { high; } \\
5=\text { very high; } \\
\text { or } \\
\text { low; mid; high }\end{array}$ & Clough 1992, Reef and \\
Lovelock 2015
\end{tabular}

\section{Table 2.}

List of quantitative traits available in the dataset and respective references of trait values.

\begin{tabular}{|c|c|}
\hline $\begin{array}{l}\text { bark carbon }(\mathrm{C}) \text { content } \\
\text { per unit bark dry mass }\end{array}$ & Koch 2002 \\
\hline $\begin{array}{l}\text { bark carbon/nitrogen }(\mathrm{C} / \\
\mathrm{N}) \text { ratio }\end{array}$ & Koch 2002 \\
\hline $\begin{array}{l}\text { bark litter nitrogen }(\mathrm{N}) \\
\text { content per unit bark dry } \\
\text { mass }\end{array}$ & Nordhaus 2004 \\
\hline $\begin{array}{l}\text { bark litter carbon }(\mathrm{C}) \\
\text { content per unit bark dry } \\
\text { mass }\end{array}$ & Nordhaus 2004 \\
\hline $\begin{array}{l}\text { bark litter carbon/ } \\
\text { nitrogen }(\mathrm{C} / \mathrm{N}) \text { ratio }\end{array}$ & Nordhaus 2004 \\
\hline $\begin{array}{l}\text { bark nitrogen }(\mathrm{N}) \\
\text { content per unit bark dry } \\
\text { mass }\end{array}$ & Koch 2002 \\
\hline dispersal unit length & $\begin{array}{l}\text { Clarke et al. 2001, Duke and Jackes 1987, Giesen et al. 2007, Hogarth 1999, NParks } \\
\text { 2017, Oliveira 2005, Soepadmo et al. 2002, Van der Stocken et al. 2015, Tomlinson } 2016\end{array}$ \\
\hline
\end{tabular}




\begin{tabular}{|c|c|}
\hline $\begin{array}{l}\text { dispersal unit litter } \mathrm{C} / \mathrm{N} \\
\text { ratio }\end{array}$ & Nordhaus 2004 \\
\hline $\begin{array}{l}\text { dispersal unit litter } \\
\text { carbon }(C) \text { content per } \\
\text { unit dry mass }\end{array}$ & Nordhaus 2004 \\
\hline $\begin{array}{l}\text { dispersal unit litter } \\
\text { nitrogen }(N) \text { content per } \\
\text { unit dry mass }\end{array}$ & Nordhaus 2004, Reise 2003 \\
\hline $\begin{array}{l}\text { dispersal unit litter } \\
\text { phosphorus }(P) \text { content } \\
\text { per unit dry mass }\end{array}$ & Reise 2003 \\
\hline $\begin{array}{l}\text { dispersal unit litter } \\
\text { potassium }(\mathrm{K}) \text { content } \\
\text { per unit dry mass }\end{array}$ & Reise 2003 \\
\hline $\begin{array}{l}\text { dispersal unit litter } \\
\text { sodium }(\mathrm{Na}) \text { content per } \\
\text { unit dry mass }\end{array}$ & Reise 2003 \\
\hline dispersal unit width & Soepadmo et al. 2002 \\
\hline $\begin{array}{l}\text { flower litter carbon }(C) \\
\text { content per flower dry } \\
\text { mass }\end{array}$ & Nordhaus 2004 \\
\hline flower litter $\mathrm{CN}$ ratio & Nordhaus 2004 \\
\hline $\begin{array}{l}\text { flower litter nitrogen }(\mathrm{N}) \\
\text { content per flower dry } \\
\text { mass }\end{array}$ & Nordhaus 2004 \\
\hline $\begin{array}{l}\text { leaf acid detergent fib } \\
\text { content per unit dry } \\
\text { mass }\end{array}$ & Amiri 2014 \\
\hline leaf area & $\begin{array}{l}\text { Arrivabene et al. 2014, Ball 1988, Lin and Wang 2001, Medina and Francisco 1997, } \\
\text { Saenger and West 2016, Yuanyue et al. 2009, Medina et al. 2001, Okello et al. 2014, } \\
\text { Reise } 2003\end{array}$ \\
\hline $\begin{array}{l}\text { leaf area per leaf mass } \\
\text { (SLA) }\end{array}$ & $\begin{array}{l}\text { Choong et al. 1992, Medina and Francisco 1997, Medina et al. 2001, Arrivabene et al. } \\
\text { 2014, Ball 1988, Lin and Wang 2001, Medina and Francisco 1997, Saenger and West } \\
\text { 2016, Yuanyue et al. 2009Wang et al. } 2011\end{array}$ \\
\hline $\begin{array}{l}\text { leaf ash content per leaf } \\
\text { dry mass }\end{array}$ & Lacerda et al. 1986 \\
\hline $\begin{array}{l}\text { leaf boron }(B) \text { content } \\
\text { per leaf dry mass }\end{array}$ & Christofoletti et al. 2013 \\
\hline
\end{tabular}




\begin{tabular}{|c|c|}
\hline $\begin{array}{l}\text { leaf calcium }(\mathrm{Ca}) \\
\text { content per leaf area }\end{array}$ & Wang et al. 2011 \\
\hline $\begin{array}{l}\text { leaf calcium }(\mathrm{Ca}) \\
\text { content per leaf dry } \\
\text { mass }\end{array}$ & $\begin{array}{l}\text { Ahmed et al. 2010, Bernini et al. 2006, Christofoletti et al. 2013, Feller 1995, Medina et al. } \\
\text { 2001, Woodroffe et al. } 1988\end{array}$ \\
\hline $\begin{array}{l}\text { leaf carbon }(C) \text { content } \\
\text { per leaf dry mass }\end{array}$ & Koch 2002, Feller 1995, Medina et al. 2001, Nordhaus et al. 2011 \\
\hline $\begin{array}{l}\text { leaf carbon/nitrogen }(\mathrm{C} / \\
\mathrm{N}) \text { ratio }\end{array}$ & $\begin{array}{l}\text { Ahmed et al. 2010, Chen and Ye 2008, Koch 2002, Medina et al. 2001, Nordhaus et al. } \\
\text { 2011, Rao et al. 1994, Schmitt } 2006\end{array}$ \\
\hline $\begin{array}{l}\text { leaf cellulose content } \\
\text { per leaf dry mass }\end{array}$ & Christofoletti et al. 2013 \\
\hline $\begin{array}{l}\text { leaf chlorine }(\mathrm{Cl}) \text { content } \\
\text { per leaf dry mass }\end{array}$ & Lacerda et al. 1986, Tong et al. 2006 \\
\hline $\begin{array}{l}\text { leaf copper }(\mathrm{Cu}) \text { content } \\
\text { per leaf dry mass }\end{array}$ & Bernini et al. 2006, Feller 1995, Christofoletti et al. 2013 \\
\hline $\begin{array}{l}\text { leaf crude fiber content } \\
\text { per leaf dry mass }\end{array}$ & Amiri 2014,Chen and Ye 2008, Choong et al. 1992, Lacerda et al. 1986, Tong et al. 2006 \\
\hline leaf cuticula thickness & Arrivabene et al. 2014, Das and Ghose 1996 \\
\hline leaf dry mass & $\begin{array}{l}\text { Arrivabene et al. 2014, Medina et al. 2001, Saenger and West 2016, Lin and Wang 2001, } \\
\text { Zimmer (unpublished data) }\end{array}$ \\
\hline $\begin{array}{l}\text { leaf dry mass per area } \\
\text { (LMA) }\end{array}$ & $\begin{array}{l}\text { Arrivabene et al. 2014, Ball 1988, Johnstone 1981, Lin and Wang 2001, Medeiros and } \\
\text { Sampaio } 2013 \text {, Medina et al. } 2001\end{array}$ \\
\hline $\begin{array}{l}\text { leaf energy content per } \\
\text { leaf dry mass }\end{array}$ & Saenger and West 2016 \\
\hline $\begin{array}{l}\text { leaf hemi-cellulose } \\
\text { content per leaf dry } \\
\text { mass }\end{array}$ & Christofoletti et al. 2013 \\
\hline $\begin{array}{l}\text { leaf intercellular } \mathrm{CO} 2 \\
\text { concentration }\end{array}$ & Mehlig 2001 \\
\hline $\begin{array}{l}\text { leaf iron }(\mathrm{Fe}) \text { content } \\
\text { per leaf dry mass }\end{array}$ & Bernini et al. 2006, Feller 1995, Medina et al. 2001, Christofoletti et al. 2013 \\
\hline leaf length & Duke and Jackes 1987, Giesen et al. 2007, Soepadmo et al. 2002 \\
\hline leaf length/width ratio & Medina et al. 2001 \\
\hline
\end{tabular}




\begin{tabular}{|c|c|}
\hline leaf lifespan & $\begin{array}{l}\text { Burrows 2003, Duke et al. 1984, Ellison 2002, Ellison and Farnsworth 1996, Gill and } \\
\text { Tomlinson 1971, Khan et al. 2009, Lee 1991, Medeiros and Sampaio 2013, Mehlig 2001, } \\
\text { Moryia et al. 1988, Saenger and West 2016, Sharma et al. 2012, Steinke 1988, Steinke } \\
\text { and Rajh 1995, Tong et al. 2006, Wang and Lin 1999, Wang'ondu et al. 2013, Wium- } \\
\text { Andersen 1981, Wium-Andersen and Christensen } 1978\end{array}$ \\
\hline $\begin{array}{l}\text { leaf lignin content per } \\
\text { leaf dry mass }\end{array}$ & Christofoletti et al. 2013 \\
\hline $\begin{array}{l}\text { leaf litter boron }(B) \\
\text { content per leaf dry } \\
\text { mass }\end{array}$ & Christofoletti et al. 2013 \\
\hline $\begin{array}{l}\text { leaf litter calcium }(\mathrm{Ca}) \\
\text { content per leaf dry } \\
\text { mass }\end{array}$ & Christofoletti et al. 2013, Woodroffe et al. 1988 \\
\hline $\begin{array}{l}\text { leaf litter carbon }(C) \\
\text { content per leaf dry } \\
\text { mass }\end{array}$ & Herbon and Nordhaus 2013, Nordhaus 2004, Nordhaus et al. 2011 \\
\hline $\begin{array}{l}\text { leaf litter carbon/ } \\
\text { nitrogen }(\mathrm{C} / \mathrm{N}) \text { ratio }\end{array}$ & $\begin{array}{l}\text { Herbon } 2011 \text {, Herbon and Nordhaus 2013, Micheli 1993, Nordhaus 2004, Nordhaus et } \\
\text { al. 2011, Rao et al. } 1994\end{array}$ \\
\hline $\begin{array}{l}\text { leaf litter cellulose } \\
\text { content per leaf dry } \\
\text { mass }\end{array}$ & Christofoletti et al. 2013 \\
\hline $\begin{array}{l}\text { leaf litter copper }(\mathrm{Cu}) \\
\text { content per leaf dry } \\
\text { mass }\end{array}$ & Christofoletti et al. 2013 \\
\hline $\begin{array}{l}\text { leaf litter energy content } \\
\text { per leaf dry mass }\end{array}$ & Nordhaus 2004 \\
\hline $\begin{array}{l}\text { leaf litter hemi-cellulose } \\
\text { content per leaf dry } \\
\text { mass }\end{array}$ & Christofoletti et al. 2013 \\
\hline $\begin{array}{l}\text { leaf litter iron (Fe) } \\
\text { content per leaf dry } \\
\text { mass }\end{array}$ & Christofoletti et al. 2013 \\
\hline $\begin{array}{l}\text { leaf litter lignin content } \\
\text { per leaf dry mass }\end{array}$ & Christofoletti et al. 2013 \\
\hline leaf litter lignin/N ratio & Gleason and Ewel 2002 \\
\hline $\begin{array}{l}\text { leaf litter magnesium } \\
(\mathrm{Mg}) \text { content per leaf } \\
\text { dry mass }\end{array}$ & Christofoletti et al. 2013, Woodroffe et al. 1988 \\
\hline
\end{tabular}




\begin{tabular}{|c|c|}
\hline $\begin{array}{l}\text { leaf litter manganese } \\
(\mathrm{Mn}) \text { content per leaf } \\
\text { dry mass }\end{array}$ & Christofoletti et al. 2013 \\
\hline $\begin{array}{l}\text { leaf litter nitrogen }(\mathrm{N}) \\
\text { content per leaf dry } \\
\text { mass }\end{array}$ & $\begin{array}{l}\text { Christofoletti et al. 2013, Herbon and Nordhaus 2013, Nordhaus 2004, Nordhaus et al. } \\
\text { 2011, Reise 2003, Steinke et al. 1993, Woodroffe et al. } 1988\end{array}$ \\
\hline $\begin{array}{l}\text { leaf litter organic matter } \\
\text { content per leaf dry } \\
\text { mass }\end{array}$ & Micheli 1993 \\
\hline $\begin{array}{l}\text { leaf litter phenolics } \\
\text { content (polyphenol) per } \\
\text { leaf dry mass }\end{array}$ & Christofoletti et al. 2013 \\
\hline $\begin{array}{l}\text { leaf litter phosphorus } \\
(\mathrm{P}) \text { content per leaf dry } \\
\text { mass }\end{array}$ & Christofoletti et al. 2013, Reise 2003, Steinke et al. 1993, Woodroffe et al. 1988 \\
\hline $\begin{array}{l}\text { leaf litter potassium }(\mathrm{K}) \\
\text { content per leaf dry } \\
\text { mass }\end{array}$ & Christofoletti et al. 2013, Reise 2003, Steinke et al. 1993, Woodroffe et al. 1988 \\
\hline $\begin{array}{l}\text { leaf litter sodium }(\mathrm{Na}) \\
\text { content per leaf dry } \\
\text { mass }\end{array}$ & Reise 2003, Woodroffe et al. 1988 \\
\hline $\begin{array}{l}\text { leaf litter sulphur (S) } \\
\text { content per leaf dry } \\
\text { mass }\end{array}$ & Christofoletti et al. 2013 \\
\hline $\begin{array}{l}\text { leaf litter tannins content } \\
\text { per leaf dry mass }\end{array}$ & Micheli 1993, Steinke et al. 1993 \\
\hline leaf litter toughness & Micheli 1993 \\
\hline $\begin{array}{l}\text { leaf litter water content } \\
\text { per leaf dry mass }\end{array}$ & Micheli 1993 \\
\hline $\begin{array}{l}\text { leaf litter zinc }(\mathrm{Zn}) \\
\text { content per leaf dry } \\
\text { mass }\end{array}$ & Christofoletti et al. 2013 \\
\hline $\begin{array}{l}\text { leaf magnesium (Mg) } \\
\text { content per leaf dry } \\
\text { mass }\end{array}$ & $\begin{array}{l}\text { Bernini et al. 2006, Christofoletti et al. 2013, Feller 1995, Medina et al. 2001, Woodroffe } \\
\text { et al. } 1988\end{array}$ \\
\hline $\begin{array}{l}\text { leaf manganese }(\mathrm{Mn}) \\
\text { content per leaf dry } \\
\text { mass }\end{array}$ & Bernini et al. 2006, Feller 1995, Medina et al. 2001, Christofoletti et al. 2013 \\
\hline
\end{tabular}




\begin{tabular}{|c|c|}
\hline $\begin{array}{l}\text { leaf maximum water use } \\
\text { efficiency }\end{array}$ & Mehlig 2001 \\
\hline $\begin{array}{l}\text { leaf nitrate (NO3-) } \\
\text { content per leaf dry } \\
\text { mass }\end{array}$ & Koch 2002 \\
\hline $\begin{array}{l}\text { leaf nitrogen }(\mathrm{N}) \text { content } \\
\text { per leaf area }\end{array}$ & Wang et al. 2011 \\
\hline $\begin{array}{l}\text { leaf nitrogen }(\mathrm{N}) \text { content } \\
\text { per leaf dry mass }\end{array}$ & $\begin{array}{l}\text { Ahmed et al. 2010, Amiri 2014, Bernini et al. 2006, Choong et al. 1992, Feller 1995, Lin } \\
\text { et al. 2006, Lin and Lin 1985, Medina and Francisco 1997, Rao et al. 1994, Schmitt 2006, } \\
\text { Tam et al. 1995, Tong et al. 2006, Christofoletti et al. 2013, Koch 2002, Wang et al. 2011, } \\
\text { Lacerda et al. 1986,Medina et al. 2001, Nordhaus 2004, Nordhaus et al. 2011, Reise } \\
\text { 2003, Woodroffe et al. } 1988\end{array}$ \\
\hline $\begin{array}{l}\text { leaf nitrogen }(\mathrm{N}) \\
\text { retranslocation prior to } \\
\text { leaf senescence }\end{array}$ & Reise 2003 \\
\hline $\begin{array}{l}\text { leaf oxalate content per } \\
\text { leaf dry mass }\end{array}$ & Koch 2002 \\
\hline $\begin{array}{l}\text { leaf phenolics content } \\
\text { (polyphenol) per leaf dry } \\
\text { mass }\end{array}$ & Christofoletti et al. 2013 \\
\hline $\begin{array}{l}\text { leaf phosphorus }(\mathrm{P}) \\
\text { content per leaf dry } \\
\text { mass }\end{array}$ & $\begin{array}{l}\text { Ahmed et al. 2010, Bernini et al. 2006, Christofoletti et al. 2013, Feller 1995, Lin and Lin } \\
\text { 1985, Medina and Francisco 1997, Tam et al. 1995, Medina et al. 2001, Reise 2003, } \\
\text { Woodroffe et al. } 1988\end{array}$ \\
\hline $\begin{array}{l}\text { leaf phosphorus }(\mathrm{P}) \\
\text { retranslocation prior to } \\
\text { leaf senescence }\end{array}$ & Reise 2003 \\
\hline $\begin{array}{l}\text { leaf photosynthesis rate } \\
\text { per leaf area }\end{array}$ & $\begin{array}{l}\text { Chen et al. 2008, Clough and Sim 1989, Jiang et al. 2017, Li et al. 2016, Lugo et al. } \\
\text { 2007, Mehlig 2001, Nandy (Datta) et al. 2005, Sobrado } 2000\end{array}$ \\
\hline $\begin{array}{l}\text { leaf potassium }(\mathrm{K}) \\
\text { content per leaf dry } \\
\text { mass }\end{array}$ & $\begin{array}{l}\text { Ahmed et al. 2010, Bernini et al. 2006, Christofoletti et al. 2013, Feller 1995, Lin and Lin } \\
\text { 1985, Tam et al. 1995, Medina et al. 2001, Woodroffe et al. } 1988\end{array}$ \\
\hline leaf sclerophyly index & Choong et al. 1992 \\
\hline $\begin{array}{l}\text { leaf sodium }(\mathrm{Na}) \\
\text { content per leaf dry } \\
\text { mass }\end{array}$ & $\begin{array}{l}\text { Ahmed et al. 2010, Feller 1995, Lacerda et al. 1986, Tong et al. 2006, Wang et al. 2011, } \\
\text { Medina et al. 2001, Woodroffe et al. } 1988\end{array}$ \\
\hline $\begin{array}{l}\text { leaf soluble tannins per } \\
\text { leaf mass }\end{array}$ & Tong et al. 2006 \\
\hline
\end{tabular}




\begin{tabular}{|c|c|}
\hline $\begin{array}{l}\text { leaf sulphur }(\mathrm{S}) \text { content } \\
\text { per leaf dry mass }\end{array}$ & Bernini et al. 2006, Christofoletti et al. 2013, Medina et al. 2001, Koch 2002 \\
\hline leaf thickness & $\begin{array}{l}\text { Arrivabene et al. 2014, Choong et al. 1992, Das and Ghose 1996, Poompozhil and } \\
\text { Kumarasamy 2014, Saenger and West 2016, Sheue et al. 2003, Yuanyue et al. 2009, } \\
\text { Zimmer M unpubl. Data }\end{array}$ \\
\hline $\begin{array}{l}\text { leaf total aminoacid } \\
\text { content per leaf dry } \\
\text { mass }\end{array}$ & Koch 2002 \\
\hline $\begin{array}{l}\text { leaf total carbohydrates } \\
\text { per leaf dry mass }\end{array}$ & Lacerda et al. 1986, Tong et al. 2006 \\
\hline $\begin{array}{l}\text { leaf total organic carbon } \\
\text { per leaf dry mass }\end{array}$ & Schmitt 2006 \\
\hline leaf toughness & Choong et al. 1992, Zimmer M unpubl. data \\
\hline $\begin{array}{l}\text { leaf transpiration rate } \\
\text { per leaf area }\end{array}$ & Mehlig 2001, Nandy (Datta) et al. 2005 \\
\hline $\begin{array}{l}\text { leaf water content per } \\
\text { leaf area }\end{array}$ & Ball 1988, Okello et al. 2014, Wang et al. 2011 \\
\hline $\begin{array}{l}\text { leaf water content per } \\
\text { leaf dry mass }\end{array}$ & $\begin{array}{l}\text { Ball 1988, Chen and Ye 2008, Choong et al. 1992, Feller 1995, Lacerda et al. 1986, } \\
\text { Saenger and West 2016, Tong et al. } 2006\end{array}$ \\
\hline $\begin{array}{l}\text { leaf zinc }(Z n) \text { content } \\
\text { per leaf dry mass }\end{array}$ & Bernini et al. 2006, Feller 1995, Christofoletti et al. 2013 \\
\hline maximum salinity & Smith 1992 \\
\hline $\begin{array}{l}\text { plant absolute maximum } \\
\text { height }\end{array}$ & $\begin{array}{l}\text { Chen and Twilley 1998, Duke and Jackes 1987, Duke et al. 2010, Ellison et al. 2010, } \\
\text { Ellison et al. 2010, FAO Ecocrop 2017, Kathiresan et al. 2010, Khan et al. 2009, NParks } \\
\text { 2017, Giesen et al. } 2007\end{array}$ \\
\hline $\begin{array}{l}\text { plant mean maximum } \\
\text { height }\end{array}$ & Duke 2010, Ellison et al. 2010Giesen et al. 2007 \\
\hline $\begin{array}{l}\text { pneumatophore } \mathrm{C} / \mathrm{N} \\
\text { ratio }\end{array}$ & Koch 2002 \\
\hline $\begin{array}{l}\text { pneumatophore carbon } \\
\text { content per unit dry } \\
\text { mass }\end{array}$ & Koch 2002 \\
\hline root $\mathrm{C} / \mathrm{N}$ ratio & Koch 2002 \\
\hline $\begin{array}{l}\text { root carbon }(\mathrm{C}) \text { content } \\
\text { per unit dry mass }\end{array}$ & Koch 2002 \\
\hline $\begin{array}{l}\text { root nitrogen }(\mathrm{N}) \text { content } \\
\text { per unit dry mass }\end{array}$ & Koch 2002 \\
\hline
\end{tabular}




\begin{tabular}{|l|l|}
\hline root porosity & Cheng et al. 2012, McKee 1996 \\
\hline root to shoot ratio & Reise 2003 \\
\hline seed air-dried mass & Royal Botanic Gardens Kew Seed Information Database (SID) 2017 \\
\hline seed C/N ratio & Nordhaus 2004 \\
\hline seed fresh mass & Royal Botanic Gardens Kew Seed Information Database (SID) 2017 \\
\hline $\begin{array}{l}\text { seed litter carbon (C) } \\
\text { content per unit dry } \\
\text { mass }\end{array}$ & Nordhaus 2004 \\
\hline seed litter nitrogen $(\mathrm{N})$ & Nordhaus 2004 \\
\hline $\begin{array}{l}\text { content per unit dry } \\
\text { mass }\end{array}$ & \\
\hline wood density & Zanne et al. 2009 \\
\hline
\end{tabular}

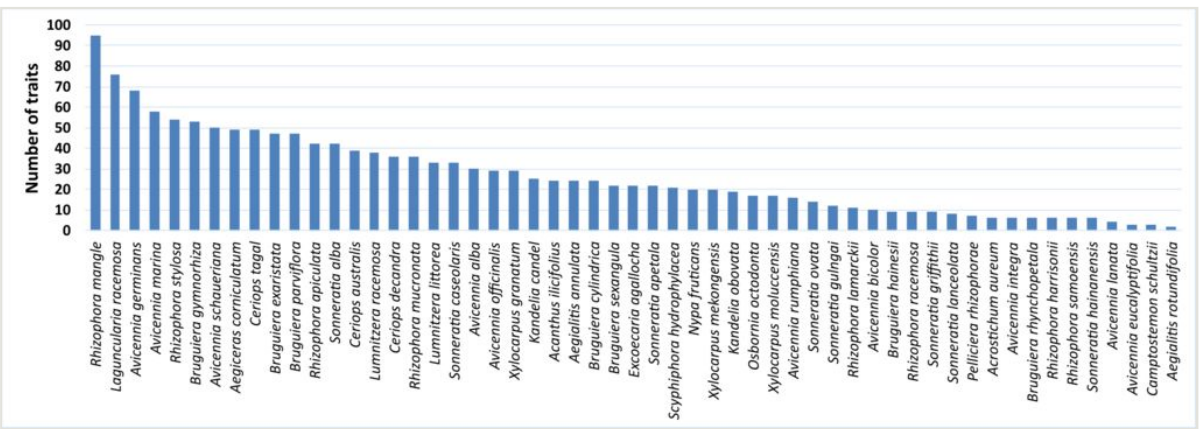

Figure 1. doi

Number of traits available per mangrove species.

\section{Remarks on data collection:}

When data was provided for young leaves and mature leaves, only mature leaves were used. When studies reported traits from the same species from different locations, all locations were considered as separate records in the database. Studies that reported a range of maximum and minimum values were also added as separate records. Leaves collected from the ground were not used for measurement of traits. For leaf litter traits, data were used where authors reported using "senescent leaves", or "yellow leaves" that could be easily detached from the trees.

To facilitate the comparison of mangrove traits with those from other studies and datasets, the same trait names were used as in the TRY Database of plant traits (KATTGE et al. 2011) whenever possible. 


\section{Usage rights}

Use license: Open Data Commons Attribution License

\section{Data resources}

Data package title: Mangrove plants traits

Resource link: https://zenodo.org/record/802990

Alternative identifiers: DOI: $10.5281 /$ zenodo.802990

Number of data sets: 1

Data set name: Mangrove plants trait dataset

Download URL: https://zenodo.org/record/802990

Data format: CSV file

\begin{tabular}{|l|l|}
\hline Column label & Column description \\
\hline Compartment & Categorical. Describes whether the trait refers to the living plant (TREE), or to the litter (LITTER). \\
\hline Organ & $\begin{array}{l}\text { Categorical. Indicates to which plant organ the trait refers (LEAF, ROOT, BARK, FLOWER, } \\
\text { DISPERSAL UNIT, SEED) or if it refers to the whole plant (TREE). }\end{array}$ \\
\hline Trait name & Trait name \\
\hline Trait value & Trait value as given in the publication \\
\hline Remarks & Any important remark about that particular value \\
\hline Plant species & Species name as given in the publication \\
\hline name & \\
\hline Trait type & Categorical. Describes whether the trait is QUANTITATIVE or QUALITATIVE \\
\hline Trait unit & Specifies the unit of quantitative traits (e.g. percentage, mg per g, mm, g) \\
\hline Source & Reference for the trait value \\
\hline Record number & Sequential record number \\
\hline
\end{tabular}




\section{References}

- $\quad$ Ahmed A, Ohlson M, Hoque S, Moula MG (2010) Chemical composition of leaves of a mangrove tree (Sonneratia apetala Buch.-Ham.) and their correlation with some soil variables. Bangladesh Journal of Botany 39: 61-69.

- Amiri F (2014) A nutritive value of Iranian mangrove ecosystems, northern part of the Persian Gulf. Natural Resources Research 23 (3): 321-330. https://doi.org/10.1007/ s11053-014-9236-x

- $\quad$ Arrivabene HP, Souza I, Oliveira Có WL, Rodella RA, Wunderlin DA, Milanez CR (2014) Functional traits of selected mangrove species in Brazil as biological indicators of different environmental conditions. Science of The Total Environment 476: 496-504. https://doi.org/10.1016/j.scitotenv.2014.01.032

- $\quad$ Ball M (1988) Ecophysiology of mangroves. Trees 2 (3): 129-142. https:// doi.org/10.1007/bf00196018

- $\quad$ Ballment E, Smith TI, Stoddart J (1988) Sibling species in the mangrove genus Ceriops (Rhizophoraceae), detected using biochemical genetics. Australian Systematic Botany 1 (4): 391. https://doi.org/10.1071/sb9880391

- Bernini E, da Silva MAB, do Carmo TMS, Cuzzuol GRF (2006) Composição química do sedimento e de folhas das espécies do manguezal do estuário do Rio São Mateus, Espírito Santo, Brasil. Revista Brasileira de Botânica 29 (4): 689-699. https:// doi.org/10.1590/s0100-84042006000400018

- Burrows DW (2003) The role of insect leaf herbivory on the mangroves Avicennia marina and Rhizophora stylosa. PhD Thesis. James Cook University URL: https:// researchonline.jcu.edu.au/1174/

- $\quad$ Chen G, Ye Y (2008) Leaf consumption by Sesarma plicata in a mangrove forest at Jiulongjiang Estuary, China. Marine Biology 154 (6): 997-1007. https://doi.org/10.1007/ s00227-008-0990-3

- $\quad$ Cheng H, Chen D, Tam NF, Chen G, Li S, Ye Z (2012) Interactions among Fe2+, S2-, and $\mathrm{Zn} 2+$ tolerance, root anatomy, and radial oxygen loss in mangrove plants. Journal of Experimental Botany 63 (7): 2619-2630. https://doi.org/10.1093/jxb/err440

- $\quad$ Chen L, Tam NY, Huang J, Zeng X, Meng X, Zhong C, Wong Y, Lin G (2008) Comparison of ecophysiological characteristics between introduced and indigenous mangrove species in China. Estuarine, Coastal and Shelf Science 79 (4): 644-652. https://doi.org/10.1016/i.ecss.2008.06.003

- $\quad$ Chen R, Twilley R (1998) A gap dynamic model of mangrove forest development along gradients of soil salinity and nutrient resources. Journal of Ecology 86 (1): 37-51. https:// doi.org/10.1046/j.1365-2745.1998.00233.x

- Choong MF, Lucas PW, Ong JSY, Pereira B, Tan HTW, Turner IM (1992) Leaf fracture toughness and sclerophylly: their correlations and ecological implications. New Phytologist 121 (4): 597-610. https://doi.org/10.1111/j.1469-8137.1992.tb01131.x

- Christofoletti R, Hattori G, Pinheiro MA (2013) Food selection by a mangrove crab: temporal changes in fasted animals. Hydrobiologia 702 (1): 63-72. https:// doi.org/10.1007/s10750-012-1307-6

- $\quad$ Clarke P, Kerrigan R, Westphal C (2001) Dispersal potential and early growth in 14 tropical mangroves: do early life history traits correlate with patterns of adult 
distribution? Journal of Ecology 89 (4): 648-659. https://doi.org/10.1046/ j.0022-0477.2001.00584.x

- Clough BF (1992) Primary productivity and growth of mangrove forests. In: Robertson Al, Alongi DM (Eds) Coastal and Estuarine Studies.Tropical Mangrove Ecosystems. https://doi.org/10.1029/ce041p0225

- $\quad$ Clough BF, Sim RG (1989) Changes in gas exchange characteristics and water use efficiency of mangroves in response to salinity and vapour pressure deficit. Oecologia 79 (1): 38-44. https://doi.org/10.1007/bf00378237

- Das S, Ghose M (1996) Anatomy of leaves of some mangroves and their associates of Sunderbans, West Bengal. Phytomorphology 46: 139-150.

- Duke N (2010) Avicennia bicolor. IUCN Red List of Threatened Species https:// doi.org/10.2305/iucn.uk.2010-2.rlts.t178847a7625682.en

- $\quad$ Duke N, Ball M, Ellison J (1998) Factors influencing biodiversity and distributional gradients in mangroves. Global Ecology and Biogeography Letters 7: 27-47. https:// doi.org/10.2307/2997695

- $\quad$ Duke N, Bunt J, Williams W (1984) Observations on the floral and vegetative phenologies of north-eastern Australian mangroves. Australian Journal of Botany 32 (1): 87. https://doi.org/10.1071/bt9840087

- $\quad$ Duke N, Kathiresan K, Salmo III SG, Fernando ES, Peras JR, Sukardjo S, Miyagi T (2010) Ceriops australis. IUCN Red List of Threatened Species https://doi.org/10.2305/ iucn.uk.2010-2.rlts.t178824a7618310.en

- $\quad$ Duke NC, Jackes BR (1987) A systematic revision of the mangrove genus Sonneratia (Sonneratiaceae) in Australasia. Blumea 32: 277-302.

- Duke NC, Meynecke JO, Dittmann S, Ellison AM, Anger K, Berger U, Cannicci S, Diele K, Ewel KC, Field CD, Koedam N, Lee SY, Marchand C, Nordhaus I, Dahdouh-Guebas F (2007) A world without mangroves? Science 317 (5834): 41b-42b. https:// doi.org/10.1126/science.317.5834.41b

- Ellison A (2002) Macroecology of mangroves: large-scale patterns and processes in tropical coastal forests. Trees 16: 181-194. https://doi.org/10.1007/s00468-001-0133-7

- Ellison A, Farnsworth E (1996) Spatial and temporal variability in growth of Rhizophora mangle saplings on coral cays: links with variation in insolation, herbivory, and local sedimentation rate. The Journal of Ecology 84 (5): 717. https://doi.org/10.2307/2261334

- Ellison A, Farnsworth E, Moore G (2010) Avicennia schaueriana. IUCN Red List of Threatened Species https://doi.org/10.2305/iucn.uk.2010-2.rlts.t178823a7617944.en

- $\quad$ Ellison J, Koedam NE, Wang Y, Primavera J, Jin Eong O, Wan-Hong Yong J, Ngoc Nam V (2010) Scyphiphora hydrophylacea. IUCN Red List of Threatened Species https:// doi.org/10.2305/iucn.uk.2010-2.rlts.t178817a7615840.en

- $\quad$ FAO Ecocrop (2017) Nypa fruticans. http://ecocrop.fao.org/ecocrop/srv/en/cropView? $i d=1543$. Accessed on: 2017-3-15.

- $\quad$ Farnsworth E (2000) The ecology and physiology of viviparous and recalcitrant seeds. Annual Review of Ecology and Systematics 31 (1): 107-138. https://doi.org/10.1146/ annurev.ecolsys.31.1.107

- $\quad$ Feller I (1995) Effects of nutrient enrichment on growth and herbivory of dwarf red mangrove (Rhizophora mangle). Ecological Monographs 65 (4): 477-505. https:// doi.org/10.2307/2963499

- $\quad$ Giesen W, Wulffraat S, Zieren M (Eds) (2007) Mangrove guidebook for southeast Asia. FAO Regional Office for Asia and the Pacific, 769 pp. [ISBN 9747946858] 
- $\quad$ Gill AM, Tomlinson PB (1971) Studies on the growth of red mangrove (Rhizophora mangle L.) 3. Phenology of the shoot. Biotropica 3 (2): 109. https://

doi.org/10.2307/2989815

- Gleason S, Ewel K (2002) Organic matter dynamics on the forest floor of a Micronesian mangrove forest: An investigation of species composition shifts. Biotropica 34 (2): 190. https://doi.org/10.1646/0006-3606(2002)034[0190:omdotf]2.0.co;2

- Herbon C, Nordhaus I (2013) Experimental determination of stable carbon and nitrogen isotope fractionation between mangrove leaves and crabs. Marine Ecology Progress Series 490: 91-105. https://doi.org/10.3354/meps10421

- Herbon CM (2011) Spatial and temporal variability in benthic food webs of the mangrove fringed Segara Anakan Lagoon in Java, Indonesia. Leibniz-Zentrum für Marine Tropenökologie, Bremen, $157 \mathrm{pp}$.

- $\quad$ Hogarth PJ (1999) The Biology of Mangroves (Biology of Habitats). New York, 228 pp. [ISBN 0198502222]

- Jiang G, Goodale UM, Liu Y, Hao G, Cao K (2017) Salt management strategy defines the stem and leaf hydraulic characteristics of six mangrove tree species. Tree Physiology 37 (3): 389-401. https://doi.org/10.1093/treephys/tpw131

- Johnstone IM (1981) Consumption of leaves by herbivores in mixed mangrove stands. Biotropica 13 (4): 252. https://doi.org/10.2307/2387803

- Kathiresan K, Salmo III SG, Fernando ES, Peras JR, Sukardjo S, Miyagi T, Ellison J, Koedam NE, Wang Y, Primavera J, Eong OJ, Wan-Hong Yong J, Ngoc Nam V (2010) Sonneratia lanceolata. IUCN Red List of Threatened Species https://doi.org/10.2305/ iucn.uk.2010-2.rlts.t178827a7619241.en

- Kattge J, Díaz S, Lavorel S, Prentice Ic, Leadley P, Bönisch G, Garnier E, Westoby M, Reich Pb, Wright lj, Cornelissen Jhc, Violle C, Harrison Sp, Van Bodegom Pm, Reichstein M, Enquist Bj, Soudzilovskaia Na, Ackerly Dd, Anand M, Atkin O, Bahn M, Baker Tr, Baldocchi D, Bekker R, Blanco Cc, Blonder B, Bond Wj, Bradstock R, Bunker De, Casanoves F, Cavender-Bares J, Chambers Jq, Chapin lii Fs, Chave J, Coomes D, Cornwell Wk, Craine Jm, Dobrin Bh, Duarte L, Durka W, Elser J, Esser G, Estiarte M, Fagan Wf, Fang J, Fernández-Méndez F, Fidelis A, Finegan B, Flores O, Ford H, Frank D, Freschet Gt, Fyllas Nm, Gallagher Rv, Green Wa, Gutierrez Ag, Hickler T, Higgins Si, Hodgson Jg, Jalili A, Jansen S, Joly Ca, Kerkhoff Aj, Kirkup D, Kitajima K, Kleyer M, Klotz S, Knops Jmh, Kramer K, Kühn I, Kurokawa H, Laughlin D, Lee Td, Leishman M, Lens F, Lenz T, Lewis SI, Lloyd J, Llusià J, Louault F, Ma S, Mahecha Md, Manning P, Massad T, Medlyn Be, Messier J, Moles At, Müller Sc, Nadrowski K, Naeem S, Niinemets Ü, Nöllert S, Nüske A, Ogaya R, Oleksyn J, Onipchenko Vg, Onoda Y, Ordoñez J, Overbeck G, Ozinga Wa, Patiño S, Paula S, Pausas Jg, Peñuelas J, Phillips Ol, Pillar V, Poorter H, Poorter L, Poschlod P, Prinzing A, Proulx R, Rammig A, Reinsch S, Reu B, Sack L, Salgado-Negret B, Sardans J, Shiodera S, Shipley B, Siefert A, Sosinski E, Soussana J-, Swaine E, Swenson N, Thompson K, Thornton P, Waldram M, Weiher E, White M, White S, Wright Sj, Yguel B, Zaehle S, Zanne Ae, Wirth C (2011) TRY - a global database of plant traits. Global Change Biology 17 (9): 2905-2935.

https://doi.org/10.1111/j.1365-2486.2011.02451.x

- Khan MN, Suwa R, Hagihara A (2009) Biomass and aboveground net primary production in a subtropical mangrove stand of Kandelia obovata (S., L.) Yong at Manko Wetland, Okinawa, Japan. Wetlands Ecology and Management 17 (6): 585-599. https:// doi.org/10.1007/s11273-009-9136-8 
- $\quad$ Koch BP (2002) Organic matter pathways in a mangrove system in northern Brazil: chemical tracers of major sources under the influence of sedimentation and biological degradation. Zentrum für Marine Tropenökologie, Bremen, 109 pp.

- Lacerda LD, Rezende CE, José DMV, Francisco MCF (1986) Metallic composition of mangrove leaves from the southeastern Brazilian coast. Revista Brasileira de Biologia 46 (2): 395-399.

- Lee SY (1991) Herbivory as an ecological process in a Kandelia candel (Rhizophoraceae) mangal in Hong Kong. Journal of Tropical Ecology 7 (3): 337-348. https://doi.org/10.1017/s0266467400005605

- $\quad$ Li F, Zan Q, Hu Z, Shin PS, Cheung S, Wong Y, Tam NF, Lei A (2016) Are photosynthetic characteristics and energetic cost Important invasive traits for alien Sonneratia species in South China? PLOS ONE 11 (6): e0157169. https:// doi.org/10.1371/journal.pone.0157169

- $\quad$ Lin P, Lin GH (1985) Studies on the mangrove ecosystem of the Jiulong Jiang River estuary in China IV. The accumulation and biological cycle of nitrogen and phosphorus elements in the Kandelia candel community. Acta Phytoecologica et Geobotanica Sinica 9: 21-31.

- $\quad$ Lin P, Wang W (2001) Changes in the leaf composition, leaf mass and leaf area during leaf senescence in three species of mangroves. Ecological Engineering 16 (3): 415-424. https://doi.org/10.1016/s0925-8574(00)00126-9

- $\quad$ Lin YM, Liu JW, Xiang P, Lin P, Ye GF, da Sternberg LSL (2006) Tannin dynamics of propagules and leaves of Kandelia candel and Bruguiera gymnorrhiza in the Jiulong River Estuary, Fujian, China. Biogeochemistry 78 (3): 343-359. https://doi.org/10.1007/ s10533-005-4427-5

- $\quad$ Lugo A, Medina E, Cuevas E, Cintrón G, Laboy Nieves E, Novelli YS (2007) Ecophysiology of a mangrove forest in Jobos Bay, Puerto Rico. Caribbean Journal of Science 43 (2): 200-219. https://doi.org/10.18475/cjos.v43i2.a6

- McKee KL (1996) Growth and physiological responses of neotropical mangrove seedlings to root zone hypoxia. Tree Physiology 16: 883-889. https://doi.org/10.1093/ treephys/16.11-12.883

- Medeiros TC, Sampaio ESB (2013) Leaf and flower formation in shoot tips of mangrove trees in Pernambuco, Brazil. Wetlands Ecology and Management 21 (3): 209-217. https://doi.org/10.1007/s11273-013-9291-9

- Medina E, Francisco M (1997) Osmolality and $\delta 13 C$ of leaf tissues of mangrove species from environments of contrasting rainfall and salinity. Estuarine, Coastal and Shelf Science 45 (3): 337-344. https://doi.org/10.1006/ecss.1996.0188

- Medina E, Giarrizzo T, Menezes M, Carvalho Lira M, Carvalho EA, Peres A, B. S, Vilhena R, Reise A, Braga Fe (2001) Mangal communities of the "Salgado Paraense": Ecological heterogeneity along the Bragança peninsula assessed through soil and leaf analyses. Amazoniana 16: 397-416.

- Mehlig U (2001) Aspects of tree primary production in an equatorial mangrove forest in Brazil. Zentrum für Marine Tropenökologie, Bremen, $155 \mathrm{pp}$.

- Micheli F (1993) Feeding ecology of mangrove crabs in North Eastern Australia: mangrove litter consumption by Sesarma messa and Sesarma smithii . Journal of Experimental Marine Biology and Ecology 171 (2): 165-186. https:// doi.org/10.1016/0022-0981(93)90002-6 
- $\quad$ Moryia H, Komiyama A, Suhardjono P, Ogino K (1988) Specific characteristics of leaf dynamics. In: Ogino K, Chihara M (Eds) Biological System of Mangroves. Ehime University Japan

- $\quad$ Nandy (Datta) P, Das S, Ghose M (2005) Relation of leaf micromorphology with photosynthesis and water efflux in some Indian mangroves. Acta Bot. Croat. 64 (2): 331-340.

- Nordhaus I (2004) Feeding ecology of the semi-terrestrial crab Ucides cordatus (Decapoda: Brachyura) in a mangrove forest in northern Brazil. Zentrum für Marine Tropenökologie, Bremen, 198 pp.

- $\quad$ Nordhaus I, Salewski T, Jennerjahn T (2011) Food preferences of mangrove crabs related to leaf nitrogen compounds in the Segara Anakan Lagoon, Java, Indonesia. Journal of Sea Research 65 (4): 414-426. https://doi.org/10.1016/i.seares.2011.03.006

- NParks (2017) Flora Fauna Web. https://florafaunaweb.nparks.gov.sg/. Accessed on: 2017-2-02.

- $\quad$ Okello J, Robert ER, Beeckman H, Kairo J, Dahdouh-Guebas F, Koedam N (2014) Effects of experimental sedimentation on the phenological dynamics and leaf traits of replanted mangroves at Gazi bay, Kenya. Ecology and Evolution 4 (16): 3187-3200. https://doi.org/10.1002/ece3.1154

- $\quad$ Oliveira VF (2005) Influência do estresse hídrico e salino na germinação de propágulos de Avicennia schaueriana Stapf e Leechman ex Moldenke e Laguncularia racemosa (L.) Gaertn. f. Instituto de Pesquisas Jardim Botânico do Rio de Janeiro, Rio de Janeiro, $8 \mathrm{pp}$.

- $\quad$ Poompozhil S, Kumarasamy D (2014) Leaf anatomical studies on some mangrove plants. Journal of Academia and Industrial Research 2: 583-589.

- Rao RG, Woitchik AF, Goeyens L, Riet Av, Kazungu J, Dehairs F (1994) Carbon, nitrogen contents and stable carbon isotope abundance in mangrove leaves from an east African coastal lagoon (Kenya). Aquatic Botany 47 (2): 175-183. https:// doi.org/10.1016/0304-3770(94)90012-4

- Reef R, Lovelock C (2015) Regulation of water balance in mangroves. Annals of Botany 115 (3): 385-395. https://doi.org/10.1093/aob/mcu174

- Reise A (2003) Estimates of biomass and productivity in fringe mangroves of NorthBrazil. Zentrum für Marine Tropenökologie https://doi.org/10.2312/ZMTCONTRIBUTIONS.2003.16

- Royal Botanic Gardens Kew Seed Information Database (SID) (2017) http:// data.kew.org/sid/

- Saenger P, West P (2016) Determinants of some leaf characteristics of Australian mangroves. Botanical Journal of the Linnean Society 180 (4): 530-541. https:// doi.org/10.1111/boj.12386

- $\quad$ Sahu SK, Singh R, Kathiresan K (2016) Multi-gene phylogenetic analysis reveals the multiple origin and evolution of mangrove physiological traits through exaptation.

Estuarine, Coastal and Shelf Science 183: 41-51. https://doi.org/10.1016/ j.ecss.2016.10.021

- Schmitt BB (2006) Characterization of organic nitrogen compounds in sediment and leaves of a mangrove ecosystem in North Brazil. Center of Marine Tropical Ecology. Bremen University, Bremen.

- Sharma S, Kamruzzaman M, Rafiqul Hoque ATM, Hagihara A (2012) Leaf phenological traits and leaf longevity of three mangrove species (Rhizophoraceae) on Okinawa 
Island, Japan. Journal of Oceanography 68 (6): 831-840. https://doi.org/10.1007/ s10872-012-0133-9

- $\quad$ Sheue C, Liu H, Yong JH (2003) Kandelia obovata (Rhizophoraceae), a new mangrove species from eastern Asia. Taxon 52 (2): 287. https://doi.org/10.2307/3647398

- Smith T (1992) Forest structure. Coastal and Estuarine Studies. https://doi.org/10.1029/ ce041p0101

- Sobrado MA (2000) Relation of water transport to leaf gas exchange properties in three mangrove species. Trees 14 (5): 258-262. https://doi.org/10.1007/s004680050011

- Soepadmo E, Saw L, Chung RCK (2002) Tree flora of Sabah and Sarawak. Malaysian Nature Society 4.

- $\quad$ Steinke TD (1988) Vegetative and floral phenology of three mangroves in Mgeni Estuary. South African Journal of Botany 54 (2): 97-102. https://doi.org/10.1016/ s0254-6299(16)31337-0

- $\quad$ Steinke TD, Rajh A (1995) Vegetative and floral phenology of the mangrove, Ceriops tagal, with observations on the reproductive behaviour of Lumnitzera racemosa, in the Mgeni Estuary. South African Journal of Botany 61 (5): 240-244. https://doi.org/10.1016/ s0254-6299(15)30529-9

- $\quad$ Steinke TD, Holland AJ, Singh Y (1993) Leaching losses during decomposition of mangrove leaf litter. South African Journal of Botany 59 (1): 21-25. https://

doi.org/10.1016/s0254-6299(16)30770-0

- $\quad$ Tam NFY, Li SH, Lan CY, Chen GZ, Li MS, Wong YS (1995) Nutrients and heavy metal contamination of plants and sediments in Futian mangrove forest. Hydrobiologia 295:

149-158. https://doi.org/10.1007/bf00029122

- $\quad$ The Plant List (2013) http://www.theplantlist.org. Accessed on: 2017-4-20.

- Tomlinson PB (1986) The Botany of Mangroves. Cambridge University Press, Cambridge, 413 pp. [ISBN 0-521-25567-8]

- Tomlinson PB (2016) The Botany of Mangroves. 2. Cambridge University Press, 436 pp. [ISBN 052146675X]

- $\quad$ Tong YF, Lee SY, Morton B (2006) The herbivore assemblage, herbivory and leaf chemistry of the mangrove Kandelia obovata in two contrasting forests in Hong Kong. Wetlands Ecology and Management 14 (1): 39-52. https://doi.org/10.1007/ s11273-005-2565-0

- Van der Stocken TV, Vanschoenwinkel B, De Ryck DR, Bouma T, Dahdouh-Guebas F, Koedam N (2015) Interaction between water and wind as a driver of passive dispersal in mangroves. PLoS ONE 10 (3): e0121593. https://doi.org/10.1371/journal.pone.0121593

- Wang L, Mu M, Li X, Lin P, Wang W (2011) Differentiation between true mangroves and mangrove associates based on leaf traits and salt contents. Journal of Plant Ecology 4 (4): 292-301. https://doi.org/10.1093/jpe/rtq008

- Wang'ondu V, Kairo J, Kinyamario J, Mwaura F, Bosire J, Dahdouh-Guebas F, Koedam N (2013) Vegetative and reproductive phenological traits of Rhizophora mucronata Lamk. and Sonneratia alba Sm. Flora - Morphology, Distribution, Functional Ecology of Plants 208: 522-531. https://doi.org/10.1016/j.flora.2013.08.004

- Wang W, Lin P (1999) Transfer of salt and nutrients in Bruguiera gymnorrhiza leaves during development and senescence. Mangroves and Salt Marshes 3 (1): 1-7. https:// doi.org/10.1023/a:1009937628112 
- Wang W, Yan Z, You S, Zhang Y, Chen L, Lin G (2011) Mangroves: obligate or facultative halophytes? A review. Trees 25 (6): 953-963. https://doi.org/10.1007/ s00468-011-0570-X

- Wium-Andersen S (1981) Seasonal growth of mangrove trees in Southern Thailand. III. Phenology of Rhizophora mucronata Lamk. and Scyphiphora hydrophyllacea Gaertn. Aquatic Botany 10: 371-376. https://doi.org/10.1016/0304-3770(81)90035-8

- Wium-Andersen S, Christensen B (1978) Seasonal growth of mangrove trees in southern Thailand. II. Phenology of Bruguiera cylindrica, Ceriops tagal, Lumnitzera littorea and Avicennia marina . Aquatic Botany 5: 383-390. https:// doi.org/10.1016/0304-3770(78)90078-5

- Woodroffe CD, Bardsley KN, Ward PJ, Hanley JR (1988) Production of mangrove litter in a macrotidal embayment, Darwin Harbour, N.T., Australia. Estuarine, Coastal and Shelf Science 26 (6): 581-598. https://doi.org/10.1016/0272-7714(88)90035-2

- Yuanyue L, Zhongbao L, Peng L (2009) The Study on the Leaf Anatomy of Some Mangrove Species of CHINA. 2009 International Conference on Environmental Science and Information Application Technology https://doi.org/10.1109/esiat.2009.397

- Zanne AE, Lopez-Gonzalez G, Coomes DA, llic J, Jansen S, Lewis SL, Miller RB, Swenson NG, Wiemann MC, Chave J (2009) Global Wood Density Database. Dryad Digital Repository https://doi.org/10.5061/DRYAD.234/1

\section{Supplementary material}

\section{Suppl. material 1: Matrix of traits per species showing the number of records per each combination.}

Authors: Aline Ferreira Quadros, Martin Zimmer

Data type: phylogenetic

Filename: Quadros and Zimmer Table.xlsx - Download file (34.25 kb) 